DRAFT VERSION OCTOBER 31, 2018

Preprint typeset using $\mathrm{LT}_{\mathrm{E}} \mathrm{X}$ style emulateapj v. 2/19/04

\title{
DISCOVERY OF THE WIDEST VERY LOW MASS BINARY
}

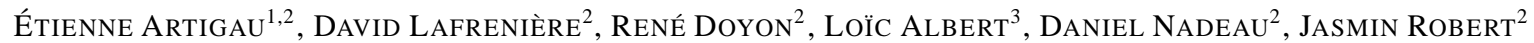 \\ Draft version October 31, 2018
}

\begin{abstract}
We report the discovery of a very low mass binary system (primary mass $<0.1 \mathrm{M}_{\odot}$ ) with a projected separation of $\sim 5100 \mathrm{AU}$, more than twice that of the widest previously known system. A spectrum covering the 1-2.5 $\mu \mathrm{m}$ wavelength interval at $R \sim 1700$ is presented for each component. Analysis of the spectra indicates spectral types of M6.5V and M8V, and the photometric distance of the system is $\sim 62 \mathrm{pc}$. Given that previous studies have established that no more than $1 \%$ of very low mass binary systems have orbits larger than 20 AU, the existence of such a wide system has a bearing on very low mass star formation and evolution models.
\end{abstract}

Subject headings: binaries: general — stars: low-mass, brown dwarfs — stars: individual (2MASS J012655.49-502238.8, 2MASS J012702.83-502321.1)

\section{INTRODUCTION}

A large fraction of the stars in the Galaxy reside in multiple systems. Generally, higher multiplicity fractions are found as the stellar mass increases, with a binarity fraction reaching $\sim 80 \%$ for B stars (Kouwenhoven et al. 2005). At the other end of the distribution, surveys of very low mass (VLM, mass $<0.1 \mathrm{M}_{\odot}$ ) stars and brown dwarfs (BDs), both in the field and in star forming regions, have found a binary fraction of $\sim 20 \%$ (Burgasser et al. 2007); corrections for incompleteness of tight systems could revise this number to a higher value.

There are important differences between the VLM binary systems and their more massive counterparts, possibly reflecting differences in their formation mechanism. The mass ratio distribution of VLM binaries is strongly peaked at unity (Burgasser et al. 2007), whereas it is more evenly distributed between 0 and 1 for more massive stars. The results of Duquennoy \& Mayor (1991) indicate that around 50\% of solar-type binary systems have separations larger than $\sim 40 \mathrm{AU}$, and that these systems can have separations as large as $\sim 20000 \mathrm{AU}$. On the other hand, small separations are strongly favored among VLM binary systems, less than $1 \%$ of which have separations larger than $20 \mathrm{AU}$ (Burgasser et al. 2007).

To date, only five VLM systems with separation > $50 \mathrm{AU}$ are known, two in the field, Koenigstuhl $1 \mathrm{AB}$ (1800 AU) and DENIS J055146.0-443412.2AB (220 AU) (Billères et al. 2005; Caballero 2007), and three in star forming regions: 2MASS J1623361-240221 (212 AU), 2MASS J1101192-773238AB (242 AU), and 2MASS J1622252240514 (243 AU) (Jayawardhana \& Ivanov 2006; Close et al. 2006; Luhman 2004). A possible binary system having a separation $\sim 1700 \mathrm{AU}$ was recently identified in the $\sigma$ Orionis cluster (Caballero et al. 2006), but this system lacks proper motion (PM) confirmation of physical association. In this Letter we report the identification of 2MASS J012655.49502238.8 and 2MASS J012702.83-502321.1 as part of a VLM binary system (hereafter referred to as 2M0126AB) with a projected separation of $\sim 5100$ AU.

\footnotetext{
${ }^{1}$ Gemini Observatory, Southern Operations Center, Association of Universities for Research in Astronomy, Inc., Casilla 603, La Serena, Chile

${ }_{2}$ Département de Physique and Observatoire du Mont Mégantic, Université de Montréal, C.P. 6128, Succ. Centre-Ville, Montréal, QC, H3C 3J7, Canada

3 Canada-France-Hawaii Telescope Corporation, 65-1238 Mamalahoa Highway, Kamuela, HI 96743, USA

Electronic address: eartigau@gemini.edu
}

\section{DISCOVERY OF THE SYSTEM AND OBSERVATIONS}

The system was found by comparing the positions of the 2MASS point source catalogue (Cutri et al. 2003) with those of the I-band Digitized Sky Survey images to search for rel-

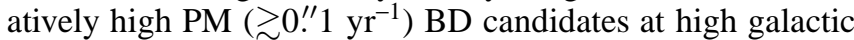
latitudes $\left(|b|>30^{\circ}\right)$. Among the sample of candidates found, the two sources mentioned above appeared to share the same PM. This was further confirmed using $J$-band observations obtained on 2006 June 21 with the Observatoire du Mont Mégantic wide field near-infrared camera CPAPIR (Artigau et al., in preparation), currently installed at the CTIO $1.5-\mathrm{m}$ telescope. The near-infrared colors (Table 1) of both components are indicative of late-M dwarfs (Leggett et al. 2002) and their apparent magnitudes place them at a similar distance, suggesting a physical association (see $\$ 4$ ).

We obtained near-infrared spectroscopic observations ( $\mathrm{R} \sim 1700$ ) of 2MASS J012655.49-502238.8 (component A) and 2MASS J012702.83-502321.1 (component B) with the GNIRS spectrograph (Elias et al. 2006) at the Gemini South telescope on 2006 August 11 (see Figure 1) with a 0." 3 slit

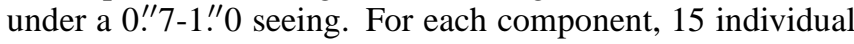
spectra were obtained for a total integration time of $15 \mathrm{~min}$ utes, and their median combination has a signal-to-noise ratio of about 30 per resolution element ( 2 pixels at $\mathrm{R} \sim 1700$ ) in the $J, H$, and $K$ bands. The A0 star HD8341 was observed for telluric absorption calibration.

\section{PROPERTIES OF THE COMPONENTS}

A summary of all measurements and derived parameters is presented in Table 1. The proper motion of each component was calculated from their positions in the SuperCosmos Sky Survey (SSS) I-band catalog (Hambly et al. 2001), the 2MASS PSC, and the CPAPIR observations; the corresponding epochs are 1983.661, 1999.778, and 2006.469. A

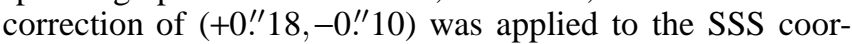
dinates to account for a local systematic offset with respect to the 2MASS PSC, as determined from the comparison of $\sim 200$ stars located within $15^{\prime}$ from $2 \mathrm{M} 0126 \mathrm{AB}$. The astrometry of the CPAPIR observations was calibrated by using the 2MASS PSC as a reference frame, and the relative astrometric uncertainty after calibration was found to be $0 .{ }^{\prime \prime} 16$. The uncertainties on the SSS coordinates $\left(0\right.$.' $\left.^{\prime} 15,0 .{ }^{\prime \prime} 3\right)$ and the 2 MASS coordinates $\left(0 .^{\prime \prime} 07,0 .^{\prime \prime} 07\right)$ were estimated from Hambly et al. (2001) and the 2MASS PSC, respectively. An error-weighted linear regression was performed to obtain the PMs and the 


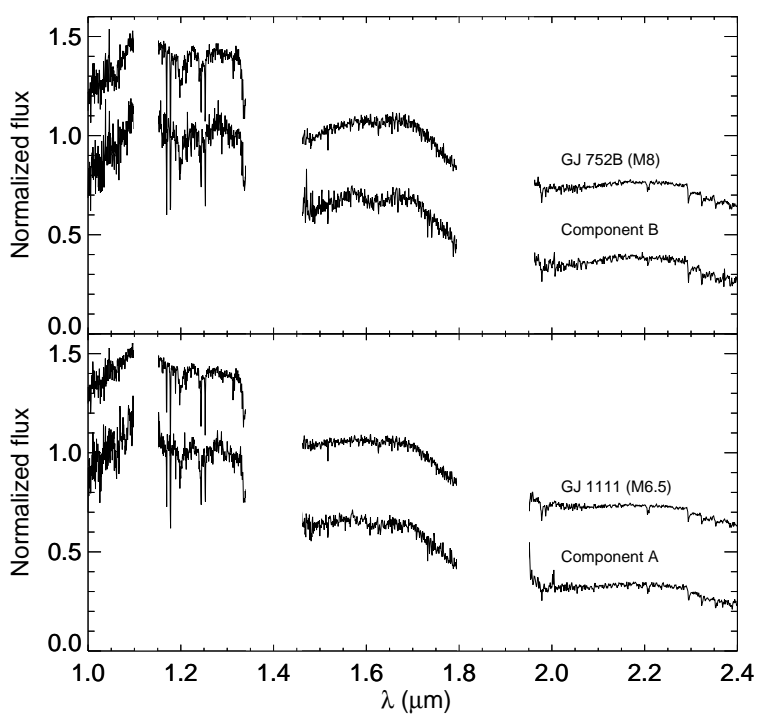

FIG. 1.- Bottom panel shows the spectrum of component A with the spectrum of an M6.5 dwarf for comparison. The top panel shows the spectrum of B with the spectrum of an M8 dwarf. All spectra are normalized to their mean in the range 1.2-1.3 $\mu \mathrm{m}$ and the spectra of comparison dwarfs have been offset by +0.4 . Spectral regions strongly affected by telluric absorption are masked out. The spike-like structure at $2.02 \mu \mathrm{m}$ in the spectrum of both $\mathrm{A}$ and $\mathrm{B}$ is most likely due to an imperfect correction of a narrow telluric absorption band. The comparison spectra are from Rayner et al. (in preparation) and were obtained with SpeX at the IRTF.

corresponding uncertainties. The PM values found for $\mathrm{A}$ and $\mathrm{B}$, respectively $131 \pm 9$ and $135 \pm 9$ mas/yr in right ascension and $-53 \pm 15$ and $-47 \pm 15$ mas/yr in declination, agree within $1 \sigma$. Although the available data make it difficult to quantify, one may assume that part of the uncertainty on the individual PMs arises from calibration errors common to both objects, so that the uncertainties on the difference in PM may be smaller than indicated by the above values.

The spectral types were derived primarily from crosscorrelation of both spectra with those of known M5-M9 dwarfs obtained with SpeX at the IRTF $^{4}$ (Rayner et al., in preparation). The wavelength intervals used for the crosscorrelation were: $1.0-1.12,1.18-1.35,1.48-1.8$, and 1.95-2.4 $\mu \mathrm{m}$. Spectral types of M6.5-M7 and M8 were found for components $\mathrm{A}$ and $\mathrm{B}$ respectively (see Figure 1). The spectral indices defined in McLean et al. (2003) yield spectral types of M6.5-M9 and M6.5-L0.5 for A and B respectively, consistent with the previous determination. The relatively short wavelength intervals $(0.004 \mu \mathrm{m})$ used for calculating these indices combined with the modest signal-to-noise ratio of our spectra explain the larger uncertainty on the spectral types using this scheme. The equivalent widths (EWs) of the KI lines at $1.169,1.177,1.244$, and $1.253 \mu \mathrm{m}$ for components $\mathrm{A}$ and $\mathrm{B}$ (see Table 1) are consistent with those of M6-M6.5 and M7-M9 dwarfs respectively, based on Figure 14 and 15 of McLean et al. (2003). Considering all of these measurements, we adopt a spectral type of M6.5V for A and M8V for B with an uncertainty of \pm 0.5 .

Photometric distances of $63 \pm 5 \mathrm{pc}$ and $61 \pm 6 \mathrm{pc}$ are calculated for A and B respectively from comparison of their $J H K_{S}$ magnitudes with the absolute magnitudes of other dwarfs of the same spectral types with known trigonometric parallaxes.

\footnotetext{
${ }^{4}$ http://irtfweb.ifa.hawaii.edu/ spex/spexlibrary/IRTFlibrary.html.
}

TABLE 1

PARAMETERS OF 2MASS J0126AB

\begin{tabular}{|c|c|c|}
\hline Parameter & $\mathrm{A}$ & $\mathrm{B}$ \\
\hline 2MASS designation & J012655.49-502238.8 & $012702.83-502321.1$ \\
\hline Angular separation & \multicolumn{2}{|c|}{$81^{\prime \prime} 86 \pm 0$ ! $^{\prime \prime} 10$} \\
\hline$\mu_{\alpha} \cos \delta^{\mathrm{a}}$ & $131 \pm 9 \mathrm{mas} / \mathrm{yr}$ & $135 \pm 9 \mathrm{mas} / \mathrm{yr}$ \\
\hline$\mu_{\delta}^{\mathrm{a}}$ & $-53 \pm 15 \mathrm{mas} / \mathrm{yr}$ & $-47 \pm 15 \mathrm{mas} / \mathrm{yr}$ \\
\hline$V^{\mathrm{b}}$ & 21.8 & 22.2 \\
\hline$I^{\mathrm{c}}$ & $17.2 \pm 0.3$ & $17.6 \pm 0.3$ \\
\hline$J^{\mathrm{d}}$ & $14.61 \pm 0.04$ & $14.81 \pm 0.05$ \\
\hline$H^{\mathrm{d}}$ & $14.05 \pm 0.05$ & $14.16 \pm 0.04$ \\
\hline$K_{s}^{\mathrm{d}}$ & $13.68 \pm 0.05$ & $13.62 \pm 0.05$ \\
\hline $\mathrm{FeH} 1.20 \mu \mathrm{m}^{\mathrm{e}}$ & $6.0 \pm 1.8 \AA$ & $14.5 \pm 1.9 \AA$ \\
\hline $\mathrm{KI} 1.25 \mu \mathrm{m}^{\mathrm{e}}$ & $9.8 \pm 1.1 \AA$ & $10.3 \pm 1.0 \AA$ \\
\hline Spectral type & $\mathrm{M} 6.5 \mathrm{~V} \pm 0.5$ & $\mathrm{M} 8 \mathrm{~V} \pm 0.5$ \\
\hline Photometric distance $\mathrm{f}^{\mathrm{f}}$ & $63 \pm 5 \mathrm{pc}$ & $61 \pm 6 \mathrm{pc}$ \\
\hline Physical separation & \multicolumn{2}{|c|}{$5100 \pm 400 \mathrm{AU}$} \\
\hline$T_{\text {eff }}^{\mathrm{g}}$ & $2670 \pm 180 \mathrm{~K}$ & $2490 \pm 180 \mathrm{~K}$ \\
\hline Mass $(>1 \mathrm{Gyr})^{\mathrm{h}}$ & $0.095 \pm 0.005 \mathrm{M}_{\odot}$ & $0.092 \pm 0.005 \mathrm{M}_{\odot}$ \\
\hline $\operatorname{Mass}(\sim 30 \mathrm{Myr})^{\mathrm{h}}$ & $0.020 \pm 0.003 \mathrm{M}_{\odot}$ & $0.019 \pm 0.003 \mathrm{M}_{\odot}$ \\
\hline
\end{tabular}

${ }^{\text {a }}$ Calculated using the SuperCosmos Sky Survey $I$-band positions (epoch 1983.661), the 2MASS point source catalogue position (epoch 1999.778), and our measurements made with CPAPIR (epoch 2006.469).

${ }^{\mathrm{b}}$ Assuming $V-I=4.6$ for both objects (see Dahn et al. 2002, fig 3)

${ }^{\mathrm{c}}$ From the SuperCosmos Sky Survey catalogue.

${ }^{\mathrm{d}}$ From the 2MASS point source catalogue.

${ }^{\mathrm{e}}$ Spectral indices defined in Gorlova et al. (2003)

${ }^{\mathrm{f}}$ Determined by comparing the $J, H$, and $K_{S}$ magnitudes of A and B with the absolute magnitudes of eight M6.5V-M7V and four M8V dwarfs with known trigonometric parallaxes; the quoted uncertainties correspond to the dispersions of the distances obtained.

${ }^{\mathrm{g}}$ Spectral-type $-T_{\text {eff }}$ relation of Golimowski et al. (2004).

${ }^{\mathrm{h}}$ Determined by minimizing the sum of the square difference between the absolute $J, H$, and $K_{s}$ magnitudes of $2 \mathrm{M} 0126 \mathrm{AB}$ and evolution models (Chabrier et al. 2000). The quoted uncertainties are derived from uncertainties in the photometric distance.

Using the spectral type-effective temperature $\left(T_{\text {eff }}\right)$ relation of Golimowski et al. (2004), we infer $T_{e f f}=2670 \pm 180 \mathrm{~K}$ for A and $T_{e f f}=2490 \pm 180 \mathrm{~K}$ for B. According to evolution models (Chabrier et al. 2000), such temperatures imply masses below $0.1 \mathrm{M}_{\odot}$, irrespective of the system age.

\section{PROBABILITY OF RANDOM ALIGNMENT}

Assuming a late-M dwarf spatial density of $2.2 \times 10^{-3} \mathrm{pc}^{-3}$ per $I$-band magnitude interval (Phan-Bao et al. 2003), there should be $\sim 2700$ M6-M8 dwarfs $\left(12.5<\mathrm{M}_{I}<14.5\right)$ between 55 and $68 \mathrm{pc}$ from the Sun. Considering that an $82^{\prime \prime}$ radius disk occupies $3.95 \times 10^{-8}$ of the sky, and assuming an isotropic distribution of late-Ms out to $68 \mathrm{pc}$, the probability of finding at least one such pair of objects over the whole sky from chance alignment is 0.15 , regardless of their apparent proper motion. A Monte Carlo calculation was performed to determine the probability that two $M$ dwarfs located in the direction of $2 \mathrm{M} 0126 \mathrm{AB}$ have PMs larger than $0 .{ }^{\prime \prime} 1 \mathrm{yr}^{-1}$ that agree with each other within our uncertainties at the $2 \sigma$ level. Using the observed dispersions in $(U, V, W)$ of late-M dwarfs in the Solar neighborhood (Bochanski et al. 2005), including the disk and halo components, and converting these velocities into PMs at the position of $2 \mathrm{M} 0126 \mathrm{AB}$, the probability is $1.4 \times 10^{-2}$. Thus, the combined probability of finding a pair of late-M dwarfs within $82^{\prime \prime}$ of each other and sharing the same proper motion within our uncertainties is $2 \times 10^{-3}$. Therefore, $2 \mathrm{M} 0126 \mathrm{AB}$ is very likely a bound system rather than a random alignment of two unrelated objects. 


\section{DISCUSSION}

As shown in Gorlova et al. (2003), the EWs of FeH and KI provide a rough constraint on surface gravity. Indeed, the strength of these features in late-type $M$ dwarfs is systematically higher (by a factor 1.5-2.0) for field objects compared to young $(\lesssim 10 \mathrm{Myr}$ ) cluster members. Based on Figure 8 of Gorlova et al. (2003), the measured $\mathrm{FeH} 1.20 \mu \mathrm{m}$ and KI $1.25 \mu \mathrm{m}$ EWs of components A and B are in better agreement with those of relatively old field objects. The mass of each component can be determined from comparison of nearinfrared absolute magnitudes with evolution models (Chabrier et al. 2000) assuming an age for the system. If we suppose an age greater than $1 \mathrm{Gyr}$, as suggested by the gravity sensitive spectral indices, we derive masses of $0.095 \pm 0.005$ and $0.092 \pm 0.005 \mathrm{M}_{\odot}$ for the two components.

Although the $\mathrm{FeH}$ and KI EWs are consistent with relatively old field objects, the age of $2 \mathrm{M} 0126 \mathrm{AB}$ remains poorly constrained given the small difference in EW between field and very young dwarfs and the significant dispersion of those values (see Figure 8 of Gorlova et al. 2003). In fact, there is some evidence that $2 \mathrm{M} 0126 \mathrm{AB}$ might be part of a young association. On the sky, it lies in the core of the young ( 30 Myr) Tucana/Horologium (TH) association (Zuckerman \& Song 2004 and references therein) located at a distance of 37-66 pc, consistent with that estimated for 2M0126AB. Furthermore, both the amplitude and direction of its PM are similar to those of nearby members (see Figure 2). We note, however, that the amplitude of the PM of 2M0126AB (142 mas/yr) is slightly larger than the largest PM of nearby members (124 mas/yr). The PM dispersion of members of the association is $\sim 11 \mathrm{mas} / \mathrm{yr}$, and is representative of relatively massive members $\left(\geq 0.5 \mathrm{M}_{\odot}\right)$. The dispersion is expected to be significantly higher for lower mass members (see Figure 4 of Pinfield, Jameson \& Hodgkin 1998). An age of $30 \mathrm{Myr}$ for 2M0126AB would imply that the two components are BDs with masses of $0.020 \pm 0.003$ and $0.019 \pm 0.003 \mathrm{M}_{\odot}$. Future determinations of the trigonometric parallax, the radial velocity, the lithium abundance, the surface gravity, and the $\mathrm{H} \alpha$ or $\mathrm{X}$-ray emission of $2 \mathrm{M} 0126 \mathrm{AB}$ will help in settling this issue, and in refining the physical characterization of the system.

In the scenario where $2 \mathrm{M} 0126 \mathrm{AB}$ is a member of the $\mathrm{TH}$ association, the random alignment probability determined earlier needs to be re-evaluated; the major argument in favor of a physical association now being the relatively small angular separation of the system given the low stellar density of the association. The association has a diameter of approximately $30 \mathrm{pc}$, hence a volume of $1.4 \times 10^{4} \mathrm{pc}^{3}$. The uncertainties on the distance of both components and their angular separation place them within a volume $5.7 \times 10^{5}$ times smaller. Assuming a Kroupa three-segment IMF (Kroupa 2001), and knowing that there are 42 members of the TH association between B8 and $\mathrm{K} 5$ (roughly $4 \mathrm{M}_{\odot}$ to $0.67 \mathrm{M}_{\odot}$ ), we estimate that the association comprises 32 objects in the mass interval between $0.015 \mathrm{M}_{\odot}$ and $0.025 \mathrm{M}_{\odot}$. Given these numbers, the probability that the two components are not physically bound is only $8.7 \times 10^{-4}$. Even assuming a factor of 5 underestimate of the number of low-mass objects in the association, this probability would still be only $2 \%$.

At a distance of $62 \mathrm{pc}$, the measured angular separation of the system corresponds to a projected physical separation of $\sim 5100$ AU, more than twice that of the Koenigstuhl 1AB system (Caballero 2007) and 20 times greater than those of other known wide VLM binaries (see Figure 3). Because
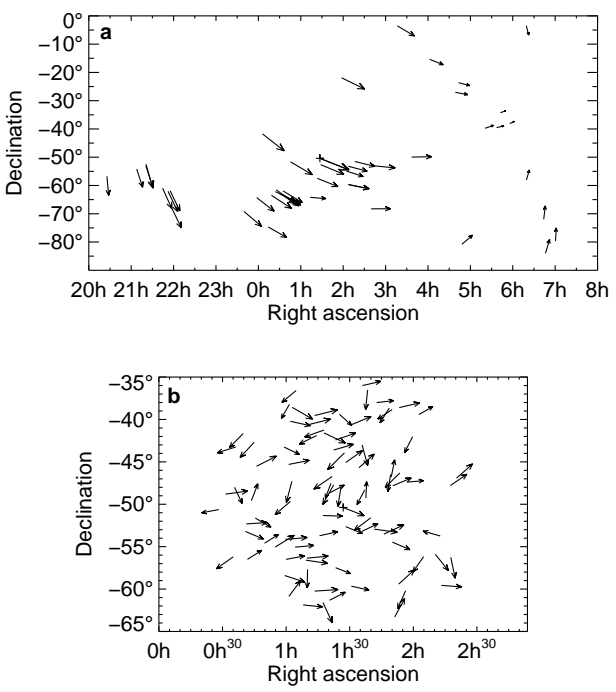

FIG. 2.- (a) The PM of all TH members listed in Zuckerman \& Song (2004) and of $2 \mathrm{M} 0126 \mathrm{AB}$ are indicated by an arrow originating from their right ascension and declination. The position of $2 \mathrm{M} 0126 \mathrm{AB}$ is indicated by a cross. $2 \mathrm{M} 0126 \mathrm{AB}$ lies in the core of $\mathrm{TH}$ and its tangential motion is very similar to that of nearby members. (b) Position and PM of 2M0126AB and of all stars in the Hipparcos catalogue located within 15 degrees of $2 \mathrm{M} 0126 \mathrm{AB}$ and with $100<\mathrm{PM}$ (mas/yr) $<150$. The position of $2 \mathrm{M} 0126 \mathrm{AB}$ is indicated by a cross. There is no overall tendency for stars in this part of the sky to move in a direction similar to that of $2 \mathrm{M} 0126 \mathrm{AB}$, or of members of $\mathrm{TH}$.

of projection effects, the real separation is expected to be, on average, 1.4 times larger (Couteau 1960) which, in the present case, would be $\sim 7000$ AU. Prior to the discovery of $2 \mathrm{M} 0126 \mathrm{AB}$, projected separations of $\sim 5000 \mathrm{AU}$ had been observed only for systems of total mass $>1 \mathrm{M}_{\odot}$. The low total mass and large separation of $2 \mathrm{M} 0126 \mathrm{AB}$ raise the question of its dynamical stability. An order of magnitude estimate of the expected survival time of 2M0126AB can be obtained from the work of Weinberg et al. (1987). Stellar and giant molecular cloud (GMC) encounters are the dominant source of disruption for low-mass binaries, and these contributions are dependent on the mass/separation ratio only, implying that the results derived for more massive binaries can be scaled to the 2M0126AB system. Given this scaling relation and Figure 2 of Weinberg et al. (1987), the stellar and GMC encounter disruption timescale of $2 \mathrm{M} 0126 \mathrm{AB}$ is $\sim 200 \mathrm{Myr}$ if it is a member of the 30 Myr old TH association or $\sim 1$ Gyr if it is a field object. The first timescale above is well beyond the age of the TH association, while the second may indicate that $2 \mathrm{M} 0126 \mathrm{AB}$ is likely younger than 2-3 Gyr. Further, the second timescale above is long enough for a significant number of similar binaries produced throughout the history of the Galaxy to have survived to this day.

The formation process of VLM stars and BDs is the subject of an active debate, partly because their mass is less than $\sim 0.7 \mathrm{M}_{\odot}$, the typical Jeans mass in molecular clouds (Meyer et al. 2000). However, it has been shown recently (Boyd \& Whitworth 2005; Padoan \& Nordlund 2004; Padoan et al. $2005)$ that turbulence can lead to fragmentation of unstable cores with masses as small as $\sim 0.003 \mathrm{M}_{\odot}$. A possibility is that VLM stars and BDs are such low-mass embryos dynamically ejected out of their feeding zone during the early stages of formation (Reipurth \& Clarke 2001). This is a popular scenario because it agrees with the observation that most VLM 


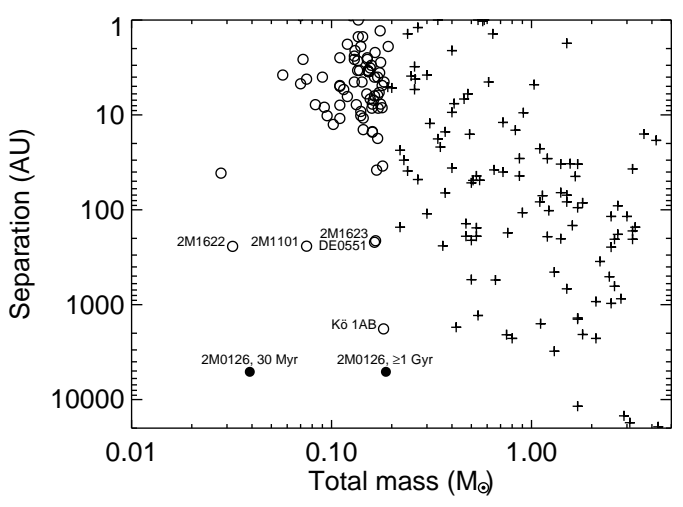

FIG. 3.- Orbital separation vs total mass of binary systems. VLM systems are shown with empty circles and stellar systems are shown with crosses. The left and right filled circles show 2M0126AB if it is aged $30 \mathrm{Myr}$ (i.e. a member of $\mathrm{TH}$ ) or $>1$ Gyr respectively. The labels refer to the large separation VLM systems mentioned in the text.

binaries have close separations, weakly bound wider binaries being easily disrupted by the ejection. Nevertheless, detailed simulations (Bate \& Bonnell 2005) of this mechanism have succeeded in producing some VLM binaries with separations $\sim 100 \mathrm{AU}$, similar to the four previously known VLM binaries mentioned above albeit with lower mass ratios. These wide binaries result when low mass cores become bound following ejection from the cluster at the same time and in the same direction. It remains to be seen whether binaries like 2M0126AB, with orbital separations $\sim 20$ times larger, can be produced by this mechanism.

Photo-evaporation of pre-stellar cores by the strong ionizing radiation of nearby massive stars (Whitworth \& Zinnecker 2004) is another possible VLM star and BD formation process. However, the presence of massive stars required for this to happen would most likely disrupt a VLM binary such as the one described here. Formation of the secondary by direct gravitational collapse within the accretion disk of the primary
(Boss 2000, 2006) is yet another possibility, but it is unlikely to form nearly equal mass binaries and would require a disk of unreasonably large spatial extent.

The formation of BDs following the encounter of protostellar disks has been shown possible in simulations (Shen \& Wadsley 2006). In this case, the disks are normally stable against gravitational collapse; it is only the violent collision between two disks that triggers instabilities leading to the formation of BDs. Although current simulations are not sufficient to address directly the question of wide binary BD formation, the results indicate that about one third of the BDs produced are ejected from the system, and wide binaries may result as in the ejection scenario discussed above. So far, the simulations have only produced BD mass objects and not VLM stars through this mechanism.

The authors are grateful to the anonymous referee for helpful comments that improved the quality of the paper. This work was supported in part through grants from the Natural Sciences and Engineering Research Council, Canada, and from Fonds Québécois de Recherche sur la Nature et les Technologies. This publication has made use of the VLM Binaries Archive maintained by Nick Siegler at http://paperclip.as.arizona.edu/ nsiegler/VLM_binaries/. Based on observations obtained at the Gemini Observatory, which is operated by the Association of Universities for Research in Astronomy, Inc., under a cooperative agreement with the NSF on behalf of the Gemini partnership: the National Science Foundation (United States), the Particle Physics and Astronomy Research Council (United Kingdom), the National Research Council (Canada), CONICYT (Chile), the Australian Research Council (Australia), CNPq (Brazil) and CONICET (Argentina). The Digitized Sky Survey was produced at the Space Telescope Science Institute under U.S. Government grant NAG W-2166. The images of these surveys are based on photographic data obtained using the Oschin Schmidt Telescope on Palomar Mountain and the UK Schmidt Telescope.

\section{REFERENCES}

Bate, M. R. \& Bonnell, I. A. 2005, MNRAS, 356, 1201

Billères, M., Delfosse, X., Beuzit, J.-L., Forveille, T., Marchal, L., \& Martín, E. L. 2005, A\&A, 440, L55

Bochanski, J. J., Hawley, S. L., Reid, I. N., Covey, K. R., West, A. A., Tinney,

C. G., \& Gizis, J. E. 2005, AJ, 130, 1871

Boss, A. P. 2000, ApJ, 536, L101

-. 2006, ApJ, 643, 501

Boyd, D. F. A. \& Whitworth, A. P. 2005, A\&A, 430, 1059

Burgasser, A. J., Reid, I. N., Siegler, N., Close, L., Allen, P., Lowrance, P., \& Gizis, J. 2007, in Protostars and Planets V, 421

Caballero, J. A., Martin, E. L., Dobbie, P. D., \& Barrado y Navascues, D. 2006, A\&A, 460, 635

Caballero, J. A. 2007, A\&A, 462, L61

Chabrier, G., Baraffe, I., Allard, F., \& Hauschildt, P. 2000, ApJ, 542, 464

Close, L. M., et al. 2006, ArXiv Astrophysics e-prints, astro-ph/0608574

Couteau, P. 1960, Journal des Observateurs, 43, 41

Cutri, R. M. et al. 2003, 2MASS All Sky Catalog of point sources. (http://irsa.ipac.caltech.edu/applications/Gator/)

Dahn, C. C. et al. 2002, AJ, 124, 1170

Duquennoy, A. \& Mayor, M. 1991, A\&A, 248, 485

Elias, J. H., Rodgers, B., Joyce, R. R., Lazo, M., Doppmann, G., Winge, C., \& Rodríguez-Ardila, A. 2006, in Proc. SPIE, Vol. 6269, 626914.

Golimowski, D. A. et al. 2004, AJ, 127, 3516

Gorlova, N. I., Meyer, M. R., Rieke, G. H., \& Liebert, J. 2003, ApJ, 593, 1074
Hambly, N. C., Davenhall, A. C., Irwin, M. J., MacGillivray, H. T 2001, MNRAS, 326, 1315

Jayawardhana, R. \& Ivanov, V. D. 2006, Science, 313, 1279

Kouwenhoven, M. B. N., Brown, A. G. A., Zinnecker, H., Kaper, L., \& Portegies Zwart, S. F. 2005, A\&A, 430, 137

Kroupa, P. 2001, MNRAS, 322, 231

Leggett, S. K., et al. 2002, ApJ, 564, 452

Luhman, K. L. 2004, ApJ, 614, 398

McLean, I. S., McGovern, M. R., Burgasser, A. J., Kirkpatrick, J. D., Prato, L., \& Kim, S. S. 2003, ApJ, 596, 561

Meyer, M. R., Adams, F. C., Hillenbrand, L. A., Carpenter, J. M., \& Larson, R. B. 2000, Protostars and Planets IV, 121

Padoan, P., Kritsuk, A., Michael, Norman, L., \& Nordlund, Å. 2005, Memorie della Societa Astronomica Italiana, 76, 187

Padoan, P. \& Nordlund, А. 2004, ApJ, 617, 559

Phan-Bao, N., et al. 2003, A\&A, 401, 959

Pinfield, D. J., Jameson, R. F., Hodgkin, S. T. 1998, MNRAS, 299, 955

Reipurth, B. \& Clarke, C. 2001, AJ, 122, 432

Shen, S. \& Wadsley, J. 2006, ApJ, 651, L145

Weinberg, M. D., Shapiro, S. L., \& Wasserman, I. 1987, ApJ, 312, 267

Whitworth, A. P. \& Zinnecker, H. 2004, A\&A, 427, 299

Zuckerman, B. \& Song, I. 2004, ARA\&A, 42, 685 